\title{
COMPARISON OF PAIN RELIEF EFFICACY OF PLATELET-RICH PLASMA VERSUS CORTICOSTEROIDS IN KNEE OSTEOARTHRITIS
}

\author{
Amir Sohail, Ameer Yasser Zaid, Shizan Hamid Feroz, Mohammad Saeed, Syed Khurram Naseer, Muhsan Sultan \\ Combined Military Hospital Peshawar/National University of Medical Sciences (NUMS) Pakistan
}

\begin{abstract}
Objective: To compare the pain relief efficacy of platelet rich plasma injection with corticosteroid injection in knee osteoarthritis using numeric rating scale.

Study Design: Quasi experimental study.

Place and Duration of Study: Department of Anesthesia and Pain Management, Combined Military Hospital Peshawar, from Jan 2018 to Dec 2019.

Methodology: Total of 310 patients who underwent knee injection for osteoarthritis were included in this study. Patients were divided into two groups; group A and group B comprising of 155 patients each. Patients in group 'A' received intra articular corticosteroid injection while patients in group ' $\mathrm{B}$ ' received intra articular platelet rich plasma injection for knee osteoarthritis. Pain assessment via numerical rating score was done at the start of the treatment and at 6 months.

Results: In group A female to male ratio was 2.69:1 while in group B the female to male ratio was 2.78:1. Mean age of 'group $\mathrm{A}^{\prime}$ ' was $58.52 \pm 11.87$ years and that of 'group B' was $58.79 \pm 11.15$ years. Numerical rating score pre-treatment in 'group A' vs 'group B' was $8.35 \pm 1.17$ vs $8.42 \pm 1.14$. While numeric rating scale post treatment in 'group A' vs 'group B' was $5.74 \pm 1.37$ vs $4.06 \pm 1.19$, respectively with $p$-value of 0.001 , which is statistically significant.

Conclusion: Patients who received intra-articular platelet rich plasma had significantly more pain relief as compared to patients who received intra-articular steroid on numerical rating score.
\end{abstract}

Keywords: Corticosteroids, Injection, Intra-articular, Numerical rating score, Osteoarthritis knee, Platelet-rich plasma.

How to Cite This Article: Sohail A, Zaid AY, Feroz SH, Saeed M, Naseer SK, Sultan M. Comparison of Pain Relief Efficacy of Platelet-Rich Plasma Versus Corticosteroids in Knee Osteoarthritis. Pak Armed Forces Med J 2021; 71(5): 1764-1768. doi: https://doi.org/10.51253/pafmj.v71i5.4135

\footnotetext{
This is an Open Access article distributed under the terms of the Creative Commons Attribution License (https://creativecommons.org/licenses/by-nc/4.0/), which permits unrestricted use, distribution, and reproduction in any medium, provided the original work is properly cited.
}

\section{INTRODUCTION}

Osteoarthritis (OA) is a disease of joints especially bigger joints like knee. In this disease, the joint cartilage breaks down over time. This problem is among the five major causes of disability in adults. 3\% of worldwide Years Lived Disability (YLD) is associated with osteoarthritis knee. ${ }^{1}$ Knee Osteoarthritis (KOA) exhibits a strong female to male preponderance. Symptoms of knee osteoarthritis are not only limited to pain but also includes limitations on range of movements. This problem has a considerably negative impact on a person's lifestyle. As the average age has increased, the number of senior citizens is also growing, and along with obesity issues, it seems that the incidence of knee osteoarthritis has also risen. ${ }^{2}$ Osteoarthritis results from an imbalance between inflammatory, anti-inflammatory cytokines and tumor necrosis factor-1, leading to the breakdown of cartilage. ${ }^{3}$

Treatment options for KOA includes nonpharmacological approaches like weight reduction, life style modifications and various physiotherapy

Correspondence: Dr Amir Sohail, Department of Anaesthesia \& Pain Medicne, Combined Military Hospital Peshawar Pakistan

Received: 20 Apr 2020; revision received: 26 Mar 2021; accepted: 30 Mar 2021 techniques. While pharmacologic therapies, including oral, topical, strontium ranelate, IL-I receptor antagonist, antibodies nerve growth factors, intra articular corticosteroids and hyaluronic acid (HA) injections. ${ }^{4}$ The corticosteroid effects are mainly anti-inflammatory, mediated by inhibiting inflammatory cytokines like Interleukin 1a (IL-1a), Interleukin 1 (IL-1) and Tumor Necrosis Factor Alpha (TNF-a). Thus blocking the pathways leading to their destructive actions on joint cartilages. ${ }^{5}$ Corticosteroid joint injection has been used to treat OA for the last five decades and finds its recommended use in different guidelines, published by The American College of Rheumatology (ACR). ${ }^{6}$

These above-mentioned techniques mostly work as damage control strategies and sort of fire extinguishing efforts, not addressing the core issues of repair and regeneration of joint cartilage. Latest scientific work is focusing on the identification of biochemical pathways that can be specifically targeted therapeutically via biological intervention for repair of cartilage. Regenerative treatment modalities, such as the one with Platelet-Rich Plasma (PRP), have been studied in many researches. The growth factors in PRP can activate cartilage regeneration, pain reduction, 
improvement in joint mobility and resultantly the quality of life.7 Insulin-Like Growth Factor (IGF) stimulates cell proliferation, accelerating production of collagen, and causing the migration of fibroblasts. Multiple centered studies have indicated that PRP is superior in relieving pain of $\mathrm{KOA}$ patients as compared to steroids. 8,9

The objective assessment of pain after receiving intra articular injections can be easily assessed using Numerical Rating Score (NRS). ${ }^{10}$ The aim of our study was to compare pain relief using NRS scale in medium term duration using corticosteroid injection in KOA, with PRP joint injection. The results of this study will help in establishing the role of PRP in OA related joint pain for short-term duration to improve quality of life of these patients. PRP will not only help in reducing the suffering of osteoarthritic patients but will reverse the damage already done and will prevent further disease progression.

\section{METHODOLOGY}

This quasi experimental study were conducted at the department of Anesthesia and Pain Management, Combined Military Hospital Peshawar, from January 2018 to December 2019. World Health Organization (WHO) sample size calculator was used to calculate sample size of the study with $5 \%$ level of significance and $90 \%$ power of test. Anticipated population proportion 1 is $63.2 \%$ and anticipated population proportion $2-45.1 \% .^{5}$ Sample size came out to be 155 in each group and 310 in total. Lottery method sampling technique was employed.

Inclusion Criteria: Patients of both genders above 30 years of age, symptomatic knee joint for more than one year, pain NRS (NRS >6), Kellgren and Lawrence OA grade II, III, IV ${ }^{11}$ and patient's willingness.

Exclusion Criteria: Patients with inability to understand and use NRS, patients with advanced rheumatoid arthritis or any other joint deformities, high uric acid levels, on antiplatelet drugs, previous knee surgeries and history of intra-articular injection within previous six weeks.

Patients in group ' $\mathrm{A}$ ' received intra articular triamcinolone $40 \mathrm{mg}$ diluted with $4 \mathrm{ml} 0.9 \%$ normal saline (total $5 \mathrm{ml}$ ) while patients in group ' $\mathrm{B}$ ' received platelet rich plasma injection (total $5 \mathrm{ml}$ ) for KOA.

In group $\mathrm{A}, 40 \mathrm{ml}$ of blood was drawn from patients to make them blind to the study. Five $\mathrm{ml}$ of diluted $40 \mathrm{mg}$ triamcinolone was injected in group A intra-articularly. While in group B, PRP was prepared by obtaining $40 \mathrm{ml}$ of autologous blood. To prevent clotting $2 \mathrm{ml}$ of ACD-A (Anticoagulant Citrate Dextrose Solution, Solution A,) was introduced to the sample. Two centrifuge cycles were run. The first one was done at 1600 Relative Centrifugal Force (RCF) for 6 minutes to separate the erythrocytes and the second spin cycle was performed at $2000 \mathrm{RCF}$ for 6 minutes to concentrate platelets, to produce $5 \mathrm{ml}$ PRP. Activation of PRP solution was achieved by introduction of $0.5 \mathrm{ml}$ of a calcium gluconate solution $(1 \mathrm{~g} / 10 \mathrm{ml})$. PRP prepared was graded as $\mathrm{P}^{2} \times \mathrm{B}^{12}$ as per Delong (PAW) classification. The solution was injected into each patient's knee immediately after preparation. Pain assessment via NRS was done at before the treatment and at six months after start of treatment. Details of the patient along with above-mentioned data were recorded on a preformed proforma. All patients were given due respect and their comfort was taken care of during the study. In both groups ultrasound guided intra-articular injections were given after taking strict aseptic measure.

Data were entered and analyzed using SPSS20. Pain was measured using NRS scoring in both the groups. Chi-square test was calculated. The $p$-value of $\leq 0.05$ was considered statistically significant.

\section{RESULTS}

A total of 310 patients receiving knee injection for osteoarthritis were included in this study. Patients were segregated into group A and group B comprising of 155 patients each. Mean age of study population was $58.43 \pm 12.65$ year. Out of study population, 83 $(26.77 \%)$ were males and $227(73.23 \%)$ were females. Group wise demographic data of patients are represented in Table-I. Difference in mean age and gender was not statistically significant between both the groups ( $p=0.322$ and 0.629 respectively).

Table-I: Demographic comparison between the groups.

\begin{tabular}{l|c|c|c}
\hline Parameters & $\begin{array}{c}\text { Group A } \\
\text { (Corticosteroid) } \\
(\mathbf{n}=\mathbf{1 5 5})\end{array}$ & $\begin{array}{c}\text { Group B } \\
(\mathbf{P R P}) \\
(\mathbf{n}=\mathbf{1 5 5})\end{array}$ & $\boldsymbol{p}$-value \\
\hline Age in Years & $58.52 \pm 11.87$ & $58.79 \pm 11.15$ & 0.322 \\
\hline Gender & $113(72.9 \%)$ & $114(73.5 \%)$ & \multirow{2}{*}{0.629} \\
\hline Female & $42(27.1 \%)$ & $41(26.5 \%)$ & \\
\hline \multicolumn{1}{|l}{ Male } &
\end{tabular}

Patients in both the groups were analyzed for OA as per Kellgren and Lawrence Osteo-arthritis grades on x-ray as per Table-II. NRS pre-treatment had a $p$ value of 0.998 compared to a $p$-value 0.001 post treatment which was significant (Table-III). 
Table-II: Comparison between the groups with respect to kellgren and lawrence osteoarthritis grades.

\begin{tabular}{|c|c|c|c|}
\hline $\begin{array}{l}\text { Kellgren and } \\
\text { Lawrence } \\
\text { Osteoarthritis } \\
\text { Grades }\end{array}$ & $\begin{array}{c}\text { Group A } \\
\text { (corticosteroid) } \\
(\mathbf{n}=155)\end{array}$ & $\begin{array}{c}\text { Group B } \\
\text { (PRP) } \\
(n=155)\end{array}$ & $\begin{array}{c}p- \\
\text { value }\end{array}$ \\
\hline Grade-2 & $21(13.5 \%)$ & $23(14.8 \%)$ & \multirow{3}{*}{0.032} \\
\hline Grade-3 & $81(52.2 \%)$ & $78(50.3 \%)$ & \\
\hline Grade-4 & $53(34.2 \%)$ & $54(34.8 \%)$ & \\
\hline
\end{tabular}

Table-III: Numeric rating scale (NRS) comparison between the study groups.

\begin{tabular}{l|c|c|c}
\hline Parameters & $\begin{array}{c}\text { Group A } \\
\text { (Corticosteroid) } \\
\text { (n=155) }\end{array}$ & $\begin{array}{c}\text { Group B } \\
\text { (PRP) } \\
\text { (n=155) }\end{array}$ & $\begin{array}{c}p \text { - } \\
\text { value }\end{array}$ \\
\hline $\begin{array}{l}\text { Pre-Treatment } \\
\text { Numeric Rating } \\
\text { Scale }\end{array}$ & $8.35 \pm 1.17$ & $8.42 \pm 1.14$ & 0.998 \\
\hline $\begin{array}{l}\text { Post-Treatment } \\
\text { Numeric Rating } \\
\text { Scale }\end{array}$ & $5.74 \pm 1.37$ & $4.06 \pm 1.19$ & 0.001 \\
\hline
\end{tabular}

\section{DISCUSSION}

The fourth leading cause of YLD is osteoarthritis, accounting for $3 \%$ of total global YLD's. ${ }^{1} \mathrm{KOA}$ is more prevalent in females as compared to males. Symptoms of knee KOA are not limited to pain and also include physical limitations on range of movements. Osteoarthritis results from disturbances between inflammatory, anti-inflammatory cytokines and tumor necrosis factor-1. There are various treatment modalities for this disease. Pharmacological treatments include corticosteroids, and intra-articular hyaluronic acid (HA) injections. ${ }^{5}$ The results of corticosteroids are mediated by inhibiting inflammatory cytokines like IL-1a, IL-1 and TNF-a thus blocking the pathways leading to their destructive actions of joint cartilages. PRP, HA and CS have been studied for their role in pain manag-ment associated with KOA and are being used in recent years to treat KOA. ${ }^{6}$ PRP, causes cartilage to regenerate resulting in pain reduction, increased joint mobility resulting in better quality of life. ${ }^{7}$

Therefore, in our study we compared pain relief in short-medium term duration with CS injection in KOA, which is conventional method, to PRP. Total of 310 patients underwent knee injection for osteoarthritis. Patients were placed into two groups comprising of 155 patients each. Those who were in group 'A' received triamcinolone $40 \mathrm{mg}$ injection while patients in group ' $\mathrm{B}$ ' received intra articular PRP injection. NRS was compared between the two groups. NRS before treatment in 'group $\mathrm{A}^{\prime}$ (corticosteroid) vs 'group $\mathrm{B}^{\prime}$ (PRP) was $8.35 \pm 1.17$ vs $8.42 \pm 1.14$. NRS post treatment at 6 months in 'group A' vs 'group B' was $5.74 \pm$
1.37 vs $4.06 \pm 1.19$, respectively with $p$-value of 0.001 , which is statistically significant.

The findings of our study were comparable with the study conducted by Forogh et al. ${ }^{5}$ In their study, a comparison of single dose of PRP injection with that of methylprednisolone acetate was made. The results of their study concluded that PRP treatment was superior for the patient's pain and symptom relief, improving quality of life and helped in performance of activities of daily living as compared to CS. This study showed that mean severity of pain in the group receiving CS before intervention was $79.1 \pm 13.4$, It changed to $63.2 \pm$ 19.7 in scound months and $72.5 \pm 16.2$, in sixth month follow-up. The severity of pain in the group treated with PRP was $80.4 \pm 14.4$ before intervention and turned into $45.1 \pm 23.4$ in the second month follow-up and $44.6 \pm 15.6$ in the sixth month follow-up. The treatment with PRP has significantly relieved the patients' pain in two and six months follow-up $(p<0.001)$. This is comparable to our study. Findings of our study were also comparable to Huang et al. ${ }^{6}$ Their study included 120 patients, who were randomized into 3 groups. The degree of improvement was significant in all groups as observed by all scores (WOMAC, NRS) in each group compared to the pretreatment values $(p<0.05)$. The mean WOMAC scores for the IA-HA group from before treatment to $3,6,9$, and 12 months were $47.23 \pm$ $5.37,25.02 \pm 4.98,26.38 \pm 5.20,27.86 \pm 4.34$, and 30.64 \pm 8.36 , respectively. Comparable improvements were seen in the IA-CS and IA-PRP groups as well. There were no significant changes in the WOMAC scores between the 3 groups 3 months after treatment $(p>0.05)$ but IA-PRP showed a considerably lower score 6,9 and 12 months after treatment $(p<0.05)$. PRP injection into the knee for symptomatic early stages of KOA is a valid treatment option. The clinical efficacy of IA PRP is comparable to that of the IA-HA and IA CS forms after 3 months and the long-term efficacy of IA PRP is superior to IA-HA and IA-CS.

Several other studies compared the effects of IA-CS (CS) with PRP in KOA. Barac et al, 7 in his study included 53 patients (90 knees). They followed these patients up to 12 months after the last injection. Patient follow up was scheduled at 2, 6 and 12 months after the last IA injection. The different pain scores (NRS, WOMAC, IKDC and KOOS) for the Cellular Matrix (CM) Group were documented at 2, 6 and 12 months after the last injection. After a gap of two months after the last injection, there were statistically significant differences in $\mathrm{CM}$ group when compared to sodium 
hyaluronate (ArthroVisc $\left.{ }^{\circledR}, \mathrm{AV}\right)$ and sodium hyaluronate with mannitol (Ostenil ${ }^{\circ}$ Plus, OP) OP groups in NRS, WOMAC, KOOS and IKDC scores with $p<0.05$. In patients treated with CM-PR-PHA, there was statistically significant $(p<0.05)$ improvement in cartilage thickness already after 2 months and also after 6 and 12 months in medial compartment, and high statistically significant improvement $(p<0.001)$ in lateral compartments.

Meheux et al,13 examined patients with painful $\mathrm{KOA}$, and concluded that PRP showed remarkable clinical improvements up to 12 months post injection. Clinical outcomes and WOMAC scores were also considerably better after PRP as compared to HA at 3-12 months post injection. However, there is low evidence for comparing leukocyte-rich versus leukocyte-poor PRP or PRP versus steroids in this study as they provided level I significance.

Finally, results of almost majority of the studies conducted in different populations of the world in comparing pain relief with CS injection versus PRP injection in KOA have shown promising results in favor of PRP.14-16 They all agree on the basis that CS injections mostly work as damage control strategy, not addresses the core issues of repair and regeneration of joint cartilage. Current research is now focusing, to pin point main pathways that can be chosen therapeutically via biological intervention for repair and regeneration of cartilage. Currently available regenerative, minimally invasive therapies, like PRP, have been researched in a number of these studies. PRP, with its gro$w$ th factors triggers cartilage regeneration that results in reduction of pain, improved functionality of the joint and hence the quality of life. Alpha granules deliver a vast amount of growth factors from enriched platelets, such as, PDG (Plated Derived Growth Factor) which result in growth of cells, regeneration and repair of blood vessels, along with collagen production, TGF beta (Transforming Growth Factor Beta) enhances cell proliferation, and production of extracellular matrix, causing angiogenesis and resultantly healing wound healing, VEGF (Vascular Endothelial Growth Factor) causes cell division and movement of endothelial cells, FGF (Fibroblast Growth Factor) which causes proliferation, EGF (Epidermal Growth Factor) effects angiogenesis, controls changes of the extracellular matrix (ECM), and hence division and migration of fibroblasts, IGF (Insulin like Growth Factor) which stimulates cell division, speeds up production of collagen, and stimulates the movement of fibroblasts. ${ }^{17,18}$ By comparing both methods in short to medium term, duration i.e. at 2-3 months helped us to choose a PRP is a better method in not only relieving the osteoarthritis pain but also regenerating and repairing the damage done due to disease process. Here we finally recommend that PRP injection not only reduced the suffering of osteoarthritic patients but will reverse the damage already done and thus prevented further disease progression.

\section{CONCLUSION}

Patients who received intra-articular platelet rich plasma had significant more pain relief as compared to patients who received intra-articular steroid on numerical rating score.

\section{LIMITATION OF STUDY}

This study only measured a pain score on NRS and that too at 6 months duration. This limitation was most profound in shorter duration and not taking into account a further elaborate functional score like WOMAC along with some radiological modalities. This study was conducted in a limited set up in Peshawar and surrounding population so its results might not be a true representation of national or international population.

\section{Conflict of Interest: None.}

\section{Authors' Contribution}

AS: Writing original draft, AYZ: Data collection, data analysis, SHF: Data collection, data analysis, MS: Article drafting, SKN: Article drafting, MS: Literature research.

\section{REFERENCES}

1. Vamshi R, Bheemisetty V, Bollabathini R, Mahadevuni V. A prospective study of intra-articular injections of platelet rich plasma in early osteoarthritis knee joint. Int J Res Orthop 2018; 4(1): 133-140.

2. Lohmander LS, Englund M. Prevalance of knee pain and knee $\mathrm{OA}$ in southern Sweden and the proportion that seeks medical care. Rhematol 2015; 54(5): 827-835.

3. Bhagat R, Saudagar R. Osteoarthritis: pathophysiology and current treatment modalities. J Drug Deliv Ther 2019; 9(3): 661-608.

4. Wu Y, Goh EL, Wang D, Ma S. Novel treatment for osteoarthritis: an update. Open Acess Rheumatol 2018; 10(2): 135-140.

5. Forogh B, Mianehsaz E, Shoaee S, Ahadi T, Raissi GR, Sajadi S. Effect of single injection of platelet-rich plasma in comparison with corticosteroid on knee osteoarthritis: a double-blind randomized clinical trial. J Sports Med Phys Fitness 2016; 56(7-8): 901-908.

6. Huang $Y$, Liu $X, X u X$, Liu J. Intra-articular injections of plateletrich plasma, hyaluronic acid or corticosteroids for knee osteoarthritis. Orthopade 2019; 48(3): 239-247.

7. Barac B, Damjanov N, Zekovic A. The new treatment approach in knee osteoarthritis: Efficacy of cellular matrix combination of platelet rich plasma with hyaluronic acid versus two different types of hyaluronic acid (HA). Int J Clin Rheumatol 2018; 13(5): 289-295.

8. Southworth TM, Naveen NB, Tauro TM, Leong NL, Cole BJ. The use of platelet-rich plasma in symptomatic knee osteoarthritis. J Knee Surg 2019; 32(1): 37-45. 


\section{Platelet-Rich Plasma Vs Corticosteroids in Knee Osteoarthritis}

9. Uslu Guvendi E, Askin A. Comparison of efficiency between corticosteroid and platelet rich plasma injection therapies in patients with knee osteoarthritis. Arch Rheumatol 2017; 33(3): 273-281.

10. Thong ISK, Jensen MP, Miro J, Tan G. The validity of pain intensity measures: what do the NRS, VAS, VRS, and FPS-R measure?. Scand J Pain 2018; 18(1): 99-107.

11. Kohn MD, Sassoon AA, Fernando ND. Classifications in Brief: Kellgren-Lawrence Classification of Osteoarthritis. Clin Orthop Relat Res 2016; 474 (8): 1886-1893.

12. Delong JM, Russell RP, Mazzocca AD. Platelet-rich plasma: the PAW classification system. Arthros 2012; 28(7): 998-1009.

13. Meheux CJ, McCulloch PC, Lintner DM, Varner KE, Harris JD. Efficacy of intra-articular platelet-rich plasma injections in knee osteoarthritis: a systematic review. Arthros 2016; 32(3): 495-505.

14. Fernández-Cuadros ME, Pérez-Moro OS. Effectiveness of platelet-rich plasma (PRP) on pain, function and quality of life in knee osteoarthritis patients: a before-and-after study and review of the literature. MOJ Orthop Rheumatol 2018; 10(3): 202-208.
15. Forogh B, Mianehsaz E, Shoaee S, Ahadi T, Raissi GR, Sajadi S. Effect of single injection of platelet-rich plasma in comparison with corticosteroid on knee osteoarthritis: a double-blind randomized clinical trial. J Sports Med Phys Fitness 2016; 56(7-8): 901-908.

16. Kavadar G, Demircioglu DT, Celik MY, Emre TY. Effectiveness of platelet-rich plasma in the treatment of moderate knee osteoarthritis: a randomized prospective study. J Phys Ther Sci 2015; 27(12): 3863-3867.

17. Kobayashi E, Fluckiger L, Kobayashi MF, Sawada K, Sculean A, Schaller B, et al. Comparative release of growth factors from PRP, PRF, and advanced PRF. Clin Oral Invest 2016; 20(2): 23532360.

18. Schär MO, Diaz-Romero J, Kohl S, Zumstein MA, Nesic D. Platelet-rich concentrates differentially release growth factors and induce cell migration in vitro. Clin Orthop Relat Res 2015; 473(5): 1635-1643. 